\title{
Biological Properties of Saline Soils Grown with Different Sorghum Cultivars
}

\author{
K. Adilakshmi*, P. Prasuna Rani, P. Ratna Prasad and R. Lakshmipathy \\ Department of Soil Science and Agricultural Chemistry, Agricultural College (ANGRAU), \\ Bapatla-522101, India \\ *Corresponding author
}

\section{A B S T R A C T}

\section{Keywords}

Electrical conductivity, Soil bacteria, Soil fungi, Soil actinomycetes and Dehydrogenase activity

Article Info

Accepted:

16 March 2018 Available Online: 10 April 2018
A pot culture experiment was conducted to study the biological properties of saline soils grown with different cultivars of sorghum during rabi 2015-2016 at Department of Soil Science and Agricultural Chemistry, Agricultural College, Bapatla. Soils of different salinity $\left(1.5,5,8\right.$ and $12 \mathrm{dS} \mathrm{m}^{-1}$ of ECe) collected from Uppugunduru region were tested using three cultivars (Hytech, Laxmi and Mahalaxmi) in completely randomized design with factorial concept replicating thrice. The salinity levels, cultivars and their interaction significantly influenced the microbial population and enzyme activity. Considerable reduction in microbial population and enzyme activity was observed at the maximum EC tested $\left(12 \mathrm{dS} \mathrm{m}^{-1}\right)$. The interaction between the salinity and cultivars at $30 \mathrm{DAS}$, flowering and harvest revealed that the bacterial population was highest $(15.73,18.50$ and $16.33 \mathrm{X}$ $\left.10^{5} \mathrm{CFU} \mathrm{g}^{-1}\right)$ in the treatment combination of $1.5 \mathrm{dS} \mathrm{m}^{-1} \mathrm{x}$ Hytech and lowest $(8.87,10.64$ and $9.66 \mathrm{X}_{10} 0^{5} \mathrm{CFU} \mathrm{g}^{-1}$ ) in the treatment combination $12 \mathrm{dS} \mathrm{m}^{-1} \times$ Mahalaxmi. Similar trends in fungal, actinomycetes population and dehydrogenase enzyme activity were recorded at $30 \mathrm{DAS}$, flowering and harvest stages.

\section{Introduction}

The microbial communities of the soil perform a fundamental role in cycling nutrients, in the organic matter content of soil and in maintaining plant productivity. Thus it is important to understand the microbial response to environmental stress, such as high concentrations of heavy metals of salts which is also known as saline stress which can gain importance, especially in agricultural soils where the high salinity may show a negative impact on soil physical, chemical, and biological properties and can ultimately deteriorate soil quality in both ecological and agricultural aspects. Increase in salinity has shown a decrease in soil respiration rate and soil microbial biomass (Pathak and Rao, 1998). The reason for the reduced size and activity of the microbial community with increasing salinity is likely to be osmotic stress which is caused by large concentrations of salts in soil solutions (Oren, 1999). Osmotic stress usually limits microbial growth and activity in saline soil, while under sodic condition, ion toxicities and adverse $\mathrm{pH}$ conditions may also inhibit microbial growth (Rietz and Haynes, 2003). A decrease in 
microbial activities with a rise in salinity is probably one of the reasons for poor crop growth in coastal saline soils (Trapathi et al., 2006). The effect is always more pronounced in the rhizosphere according to the increase in water absorption by the plants due to transpiration. The simple explanation for this is that life in high salt concentrations has a high bio-energetic taxation, since the microorganisms need to maintain osmotic equilibrium between the cytoplasm and the surrounding medium, excluding sodium ions from inside the cell.

As a result, energy sufficient for osmo adaptation is required (Jiang et al., 2007). Specific ion toxicities (e.g. those of $\mathrm{Na}$ and Cl) may also tend to inhibit microbial growth in saline soils (Zahran, 1997). The activity of exo-cellular enzymes involved in $C(\beta-$ glucosidase) mineralization declined exponentially with increasing salinity (Rietz et al., 2001).

\section{Materials and Methods}

The experiment was conducted at Green House, Department of Soil Science and Agricultural Chemistry, Agricultural College, Bapatla, by growing three sorghum cultivars (Hytech, Laxmi and Mahalaxmi) in soils of similar characteristics but variable salinity (1.5, 5, 8 and $12 \mathrm{dS} \mathrm{m}^{-1}$ of ECe) collected from Uppugunduru region in a completely randomized design with factorial concept replicating thrice. Soil samples were collected at different stages of plant growth and are analysed for biological activity and other properties as per the following protocols.

\section{Electrical conductivity (EC)}

The electrical conductivity in saturated extract (ECe) and in 1:2.5 soil water suspension was determined by using electrical conductivity bridge CM-180 (Jackson, 1973).

\section{Enumeration of microbial population}

Bacteria, fungi and actinomycetes were estimated as per the procedures outlined by Paroda (2007).

\section{Bacteria}

The enumeration of total bacteria in fresh soil samples was carried out by following serial dilution plate count technique (Dhingra and Sinclair, 2000) using nutrient agar medium (agar agar-20 g; beef extract-3 g; peptone-5 g; water - 1 litre).

Exactly $1 \mathrm{~g}$ of soil was added to $10 \mathrm{~mL}$ of sterile water to dilute the population by 10 times, from which $0.1 \mathrm{~mL}$ was transferred to another $9.9 \mathrm{~mL}$ of sterile water and this dilution process was continued till $10^{5}$ dilutions and $0.1 \mathrm{~mL}$ of the final diluted solution was transferred on to a petri plate with nutrient agar medium and spread with ' $\mathrm{L}$ ' shaped glass rod and allowed it to grow for one and half day after which colony counts were recorded.

\section{Fungi}

The enumeration of total fungi in the fresh soil samples of all treatments was carried out by following the standard serial dilution plate technique using Martins Rose Bengal Agar for fungi (Martin, 1950). The population was expressed in terms of colony forming units per $\mathrm{g}$ of soil (CFU g ${ }^{-1}$ soil).

\section{Actinomycetes}

The enumeration of total actinomycetes in the fresh soil samples of all treatments was carried out by following the standard serial dilution plate technique using Khusters Nutrient Agar medium. The population was expressed in terms of colony forming units per $\mathrm{g}$ of soil (CFU g ${ }^{-1}$ soil). 


\section{Dehydrogenage activity}

Dehydrogenase activity in the soil sample was determined by following the procedure as described by Casida et al., (1964).

\section{Results and Discussion}

\section{Soil electrical conductivity}

The EC values in 1: 2.5 soil water suspension of the initial soil used for the present experiment with ECe (saturation extract) of $1.5,5,8$ and $12 \mathrm{dS} \mathrm{m}^{-1}$ were $0.49,1.39,2.54$ and $5.06 \mathrm{dS} \mathrm{m}^{-1}$, respectively (Table 1).

The electrical conductivity (1:2.5 soil water system) measured at different stages of crop growth viz., 30 DAS, flowering and harvest (Table 1) revealed that, the EC values observed in different growth stages at variable levels of ECe were found to be more or less maintained at 30 DAS but later declined. However the EC (1:2.5 soil water system) followed an increasing trend with increase in ECe level at all growth stages. The decrease in EC values with advancement of the crop could be attributed to the leaching of salts to deeper layers (below $15 \mathrm{~cm}$ ) due to the irrigation provided for successful growth of crop. Similar decrease in EC was also observed by Petersen (1996).

\section{Influence of Salinity on biological properties of soil}

\section{Soil bacteria}

Data pertaining to soil bacterial population measured at different growth stages of crop viz., 30 DAS, flowering and harvest as affected by salinity levels and different sorghum cultivars are presented in table 2 . The soil bacterial population was significantly influenced by the salinity levels, varieties and their interaction.
The highest soil bacterial population (15.52 X $10^{5} \mathrm{CFU} \mathrm{g}^{-1}$ ) at $30 \mathrm{DAS}$ was recorded at the lowest salinity level of $1.5 \mathrm{dS} \mathrm{m}^{-1}$, which was found to be significant over the remaining salinity levels. The lowest soil bacterial population of $9.21 \times 10^{5} \mathrm{CFU} \mathrm{g}^{-1}$ was recorded at the highest salinity level of $12 \mathrm{dS}$ $\mathrm{m}^{-1}$. Similar trend of decrease in soil bacterial population with salinity was observed at flowering and harvest stages also with the highest (17.80 and $\left.16.12 \times 10^{5} \mathrm{CFU} \mathrm{g}^{-1}\right)$ and the lowest values $\left(11.16\right.$ and $10.22 \times 10^{5} \mathrm{CFU}$ $\mathrm{g}^{-1}$ ) recorded by $1.5 \mathrm{dS} \mathrm{m}^{-1}$ and $12 \mathrm{dS} \mathrm{m}^{-1}$ salinity levels, respectively. At all the three stages each level was significantly superior over the higher levels of salinity.

At 30 DAS, flowering and at harvest the highest bacterial population of 13.08, 15.01 and $13.40 \times 10^{5} \mathrm{CFU} \mathrm{g}{ }^{-1}$, respectively was observed with cultivar Hytech whereas, the lowest bacterial population of $12.05,13.57$ and $12.79 \times 10^{5} \mathrm{CFU} \mathrm{g}{ }^{-1}$, respectively was observed with cultivar Mahalaxmi.

The interaction effect of salinity and cultivars on bacterial population was significant and the highest bacterial population was recorded in the treatment combination of $1.5 \mathrm{dS} \mathrm{m} \mathrm{m}^{-1} \mathrm{X}$ Hytech $\left(15.73,18.50\right.$ and $16.33 \times 10^{5} \mathrm{CFU} \mathrm{g}^{-}$ $\left.{ }^{1}\right)$ at 30 DAS, flowering and harvest stages whereas, the lowest bacterial population (8.87, 10.64 and $9.66 \times 10^{5} \mathrm{CFU} \mathrm{g}^{-1}$ ) was recorded by the treatment combination $12 \mathrm{dS} \mathrm{m} \mathrm{m}^{-1} \mathrm{X}$ Mahalaxmi at all stages of crop growth, respectively.

\section{Soil fungi}

The soil fungal population was significantly influenced by the salinity levels, varieties and their interaction.

The data related to soil fungi (Table 3) revealed a significant decrease in fungal population with increase in salinity from 1.5 
$\mathrm{dS} \mathrm{m} \mathrm{m}^{-1}$ to $12 \mathrm{dS} \mathrm{m}^{-1}$ at all growth stages. The highest soil fungal population of 10.82, 11.61

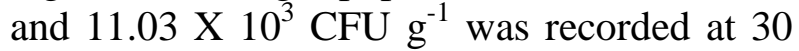
DAS, flowering and harvest, respectively with the lowest salinity level of $1.5 \mathrm{dS} \mathrm{m}^{-1}$, which was found to be significant over the remaining salinity levels. The lowest soil fungal population at all growth stages $(7.12,8.76$ and $7.96 \times 10^{3} \mathrm{CFU} \mathrm{g}^{-1}$, respectively at $30 \mathrm{DAS}$, flowering and harvest was recorded at higher salinity level of $12 \mathrm{dS} \mathrm{m}^{-1}$.

Regarding the cultivars tested, the Hytech cultivar resulted in a significantly higher fungal population over others at all growth stages. The cultivar Laxmi recorded intermediate values at all the stages and it showed a significant effect over Mahalaxmi at early stages but was comparable at harvest. The lowest fungal population was recorded by the cultivar Mahalaxmi at all stages of the crop growth.

The critical observation of data indicated that, the interaction effect of salinity and cultivars was significant on fungal population. The highest fungal population at all growth stages was recorded by the treatment combination $1.5 \mathrm{dS} \mathrm{m}^{-1} \mathrm{x}$ Hytech while the lowest population was recorded by the treatment combination $12 \mathrm{dS} \mathrm{m}^{-1} \mathrm{x}$ Mahalaxmi.

\section{Soil actinomycetes}

Data pertaining to soil actinomycetes population (X $10^{4} \quad \mathrm{CFU} \mathrm{g}^{-1}$ ) revealed a significant influence of salinity levels, varieties and their interaction are presented in table 4. In general the soil actinomycetes population decreased with increasing salt concentration.

At 30 DAS the highest soil actinomycetes population of $15.03 \times 10^{4} \mathrm{CFU} \mathrm{g}^{-1}$ was recorded at the lowest salinity level of $1.5 \mathrm{dS}$ $\mathrm{m}^{-1}$, which was found to be significant over the remaining salinity levels. The lowest soil actinomycetes population of $9.17 \times 10^{4} \mathrm{CFU}$ $\mathrm{g}^{-1}$ was recorded at higher salinity level of 12 $\mathrm{dS} \mathrm{m}^{-1}$. Similar trend of decrease in soil actinomycetes population with salinity was observed at flowering and harvest. From the data, it can be inferred that the per cent reduction in soil actinomycetes population from the lowest salinity level to the highest salinity level $\left(1.5 \mathrm{dS} \mathrm{m}^{-1}\right.$ to $\left.12 \mathrm{dS} \mathrm{m}^{-1}\right)$ was $38.99,34.33$ and 28.93 at $30 \mathrm{DAS}$, flowering and harvest, respectively.

At 30 DAS, flowering and at harvest the highest actinomycetes population of 12.70 , 14.15 and $13.51 \times 10^{4} \mathrm{CFU} \mathrm{g}^{-1}$, respectively was observed with cultivar Hytech $\left(\mathrm{V}_{1}\right)$ whereas, the lowest actinomycetes population of $12.06,13.85$ and $12.63 \times 10^{4} \mathrm{CFU} \mathrm{g}^{-1}$, respectively was observed with cultivar Mahalaxmi but at flowering the highest actinomycetes population of $14.15 \times 10^{4} \mathrm{CFU}$ $\mathrm{g}^{-1}$ was observed with cultivar Hytech. The lowest actinomycetes population was recorded by the cultivar Mahalaxmi at all stages of the crop growth.

The interactions revealed that the highest actinomycetes population was recorded in treatment combination of $1.5 \mathrm{dS} \mathrm{m}^{-1} \times$ Hytech at all growth stages. This treatment combination was found to be at par with 1.5 $\mathrm{dS} \mathrm{m} \mathrm{m}^{-1} \mathrm{x}$ Laxmi at 30 DAS and harvest and with $1.5 \mathrm{dS} \mathrm{m}^{-1} \mathrm{X}$ Laxmi and $1.5 \mathrm{dS} \mathrm{m}^{-1} \mathrm{x}$ Mahalaxmi at flowering and all other treatment combinations were inferior to other treatments. At all stages the lowest was recorded with Mahalaxmi at the highest salinity.

The perusal of data indicated that all groups of microbes studied were sensitive to salinity. However the population of microbes was found to be maximum at flowering due to active plant growth, which results in high root exudation. 
Table.1 Electrical conductivity $\left(\mathrm{dS} \mathrm{m} \mathrm{m}^{-1}\right)$ in 1:2.5 soil water system of soils at $30 \mathrm{DAS}$, flowering and at harvest stage

\begin{tabular}{|c|c|c|c|c|c|c|c|c|c|c|c|c|c|}
\hline \multirow{2}{*}{\multicolumn{2}{|c|}{$\begin{array}{l}\text { Salinity levels } \\
\quad\left(\mathrm{dS} \mathrm{\textrm {m } ^ { - 1 } )}\right.\end{array}$}} & \multirow{2}{*}{\multicolumn{4}{|c|}{ Electrical conductivity $\left(\mathrm{dS} \mathrm{m}^{-1}\right)$ at 30 DAS }} & \multicolumn{4}{|c|}{$\begin{array}{l}\text { Electrical conductivity }\left(\mathrm{dS} \mathrm{m}^{-1}\right) \text { at flowering } \\
\text { stage }\end{array}$} & \multicolumn{4}{|c|}{ Electrical conductivity $\left(\mathrm{dS} \mathrm{m}^{-1}\right)$ at harvest stage } \\
\hline & & & & & & & Sorghum & ultivars & & \multicolumn{4}{|c|}{ Sorghum cultivars } \\
\hline ECe & $\begin{array}{c}\text { EC } \\
(1: 2.5)\end{array}$ & $\begin{array}{c}\mathrm{V}_{1} \\
\text { (Hytech) }\end{array}$ & $\begin{array}{c}\mathrm{V}_{2} \\
(\text { Laxmi) }\end{array}$ & $\begin{array}{c}\mathrm{V}_{3} \\
\text { (Mahalaxmi) }\end{array}$ & Mean & $\begin{array}{c}\mathrm{V}_{1} \\
\text { (Hytech) }\end{array}$ & $\begin{array}{c}\mathbf{V}_{2} \\
(\text { Laxmi) }\end{array}$ & $\begin{array}{c}\mathrm{V}_{3} \\
\text { (Mahalaxmi) }\end{array}$ & Mean & $\begin{array}{c}\mathrm{V}_{1} \\
\text { (Hytech) }\end{array}$ & $\begin{array}{c}\mathrm{V}_{2} \\
(\text { Laxmi) }\end{array}$ & $\begin{array}{c}\mathrm{V}_{\mathbf{3}} \\
\text { (Mahalaxmi) }\end{array}$ & Mean \\
\hline $\begin{array}{c}\mathrm{S}_{1} \\
(1.5)\end{array}$ & 0.49 & 0.49 & 0.44 & 0.42 & 0.45 & 0.25 & 0.20 & 0.24 & 0.23 & 0.14 & 0.16 & 0.20 & 0.17 \\
\hline $\mathrm{S}_{2}(\mathbf{5})$ & 1.39 & 1.36 & 1.26 & 1.15 & 1.26 & 1.20 & 0.98 & 1.11 & 1.10 & 1.00 & 1.02 & 0.99 & 1.00 \\
\hline $\mathrm{S}_{3}(\mathbf{8})$ & 2.54 & 2.37 & 2.26 & 2.25 & 2.29 & 2.20 & 2.27 & 2.27 & 2.25 & 2.26 & 2.20 & 2.20 & 2.22 \\
\hline$S_{4}(12)$ & 5.06 & 4.38 & 4.43 & 4.92 & 4.58 & 4.30 & 4.20 & 4.07 & 4.19 & 4.08 & 4.17 & 4.05 & 4.10 \\
\hline \multirow{2}{*}{\multicolumn{2}{|c|}{ Mean }} & 2.15 & 2.10 & 2.19 & & 1.99 & 1.91 & 1.92 & & 1.87 & 1.89 & 1.86 & \\
\hline & & SEm \pm & CD (0.05) & \multicolumn{2}{|l|}{ CV\% } & SEm \pm & CD (0.05) & \multicolumn{2}{|l|}{ CV\% } & SEm \pm & CD (0.05) & \multicolumn{2}{|l|}{ CV\% } \\
\hline \multicolumn{2}{|c|}{ Factor $\mathbf{S}$} & 0.03 & 0.08 & \multirow{3}{*}{\multicolumn{2}{|c|}{3.95}} & 0.03 & 0.08 & \multirow{3}{*}{\multicolumn{2}{|c|}{4.02}} & 0.02 & 0.05 & \multirow{3}{*}{\multicolumn{2}{|c|}{2.48}} \\
\hline \multicolumn{2}{|c|}{ Factor V } & 0.02 & NS & & & 0.02 & NS & & & 0.01 & NS & & \\
\hline \multicolumn{2}{|c|}{ S X V } & 0.05 & 0.14 & & & 0.05 & 0.13 & & & 0.03 & 0.08 & & \\
\hline
\end{tabular}

Table.2 Soil bacterial population $\left(\mathrm{CFU} \mathrm{g} \mathrm{g}^{-1} \times 10^{5}\right)$ as influenced by salinity and sorghum cultivars

\begin{tabular}{|c|c|c|c|c|c|c|c|c|c|c|c|c|}
\hline \multirow{3}{*}{ 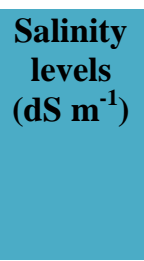 } & \multicolumn{4}{|c|}{$\begin{array}{c}\text { Soil bacterial population }\left(\mathrm{CFU} \mathrm{g}^{-1} \times 10^{5}\right) \\
\text { at } 30 \mathrm{DAS}\end{array}$} & \multicolumn{4}{|c|}{$\begin{array}{c}\left.\text { Soil bacterial population (CFU } \mathrm{g}^{-1} \times 10^{5}\right) \\
\text { at flowering stage }\end{array}$} & \multicolumn{4}{|c|}{$\begin{array}{c}\text { Soil bacterial population }\left(\mathrm{CFU} \mathrm{g}^{-1} \times 10^{5}\right) \\
\text { at harvest stage }\end{array}$} \\
\hline & \multicolumn{4}{|c|}{ Sorghum Cultivars } & \multicolumn{4}{|c|}{ Sorghum Cultivars } & \multicolumn{4}{|c|}{ Sorghum Cultivars } \\
\hline & $\begin{array}{c}\mathrm{V}_{1} \\
\text { (Hytech) }\end{array}$ & $\begin{array}{c}\mathbf{V}_{2} \\
(\text { Laxmi) }\end{array}$ & $\begin{array}{c}\mathbf{V}_{3} \\
\text { (Mahalaxmi) }\end{array}$ & Mean & V $_{1}$ (Hytech) & $\mathrm{V}_{2}$ (Laxmi) & $\mathbf{V}_{3}$ (Mahalaxmi) & Mean & $\begin{array}{c}\mathrm{V}_{1} \\
\text { (Hytech) }\end{array}$ & $\mathrm{V}_{2}$ (Laxmi) & $\mathbf{V}_{3}$ (Mahalaxmi) & Mean \\
\hline$S_{1}(1.5)$ & 15.73 & 15.63 & 15.20 & 15.52 & 18.50 & 18.19 & 16.71 & 17.80 & 16.33 & 16.03 & 16.00 & 16.12 \\
\hline$S_{2}(5)$ & 14.20 & 13.85 & 13.61 & 13.89 & 16.21 & 15.04 & 13.87 & 15.04 & 14.28 & 14.09 & 13.96 & 14.11 \\
\hline $\mathrm{S}_{3}(\mathbf{8})$ & 12.87 & 11.87 & 10.53 & 11.76 & 13.74 & 13.71 & 13.04 & 13.50 & 12.33 & 12.00 & 11.53 & 11.95 \\
\hline$S_{4}(12)$ & 9.54 & 9.22 & 8.87 & 9.21 & 11.58 & 11.27 & 10.64 & 11.16 & 10.66 & 10.33 & 9.66 & 10.22 \\
\hline Mean & 13.08 & 12.64 & 12.05 & & 15.01 & 14.55 & 13.57 & & 13.40 & 13.11 & 12.79 & \\
\hline & SEm \pm & CD (0.05) & \multicolumn{2}{|l|}{ CV\% } & SEm \pm & CD (0.05) & \multicolumn{2}{|l|}{ CV\% } & SEm \pm & CD (0.05) & \multicolumn{2}{|l|}{ CV\% } \\
\hline Factor $\mathrm{S}$ & 0.15 & 0.44 & \multirow{3}{*}{\multicolumn{2}{|c|}{3.63}} & 0.13 & 0.39 & \multirow{3}{*}{\multicolumn{2}{|c|}{2.79}} & 0.06 & 0.19 & \multirow{3}{*}{\multicolumn{2}{|c|}{1.47}} \\
\hline Factor V & 0.13 & 0.39 & & & 0.12 & 0.34 & & & 0.06 & 0.16 & & \\
\hline S X V & 0.26 & 0.77 & & & 0.23 & 0.68 & & & 0.11 & 0.32 & & \\
\hline
\end{tabular}


Table.3 Soil fungal population $\left(\mathrm{CFU} \mathrm{g} \mathrm{g}^{-1} \times 10^{3}\right)$ as influenced by salinity and sorghum cultivars

\begin{tabular}{|c|c|c|c|c|c|c|c|c|c|c|c|c|}
\hline \multirow[t]{3}{*}{$\begin{array}{l}\text { Salinity levels } \\
\quad\left(\mathrm{dS} \mathrm{m^{-1 } )}\right.\end{array}$} & \multicolumn{4}{|c|}{$\begin{array}{l}\text { Soil fungal population }\left(\mathrm{CFU} \mathrm{g}^{-1} \times \mathbf{1 0}^{3}\right) \\
\text { at } 30 \mathrm{DAS}\end{array}$} & \multicolumn{4}{|c|}{$\begin{array}{l}\text { Soil fungal population }\left(\mathrm{CFU}^{-1} \times \mathbf{1 0}^{3}\right) \\
\text { at flowering stage }\end{array}$} & \multicolumn{4}{|c|}{$\begin{array}{l}\text { Soil fungal population }\left(\mathrm{CFU} \mathrm{g}^{-1} \times \mathbf{1 0}^{3}\right) \\
\text { at harvest stage }\end{array}$} \\
\hline & \multicolumn{4}{|c|}{ Sorghum Cultivars } & \multicolumn{4}{|c|}{ Sorghum Cultivars } & \multicolumn{4}{|c|}{ Sorghum Cultivars } \\
\hline & $\begin{array}{c}\mathrm{V}_{1} \\
\text { (Hytech) }\end{array}$ & $\begin{array}{c}\mathrm{V}_{2} \\
(\mathbf{L a x m i})\end{array}$ & $\begin{array}{c}\mathrm{V}_{3} \\
\text { (Mahalaxmi) }\end{array}$ & Mean & $\begin{array}{c}\mathrm{V}_{1} \\
\text { (Hytech) }\end{array}$ & $\begin{array}{c}\mathrm{V}_{2} \\
(\mathrm{Laxmi})\end{array}$ & $\begin{array}{c}\mathrm{V}_{3} \\
\text { (Mahalaxmi) }\end{array}$ & Mean & $\begin{array}{c}\mathrm{V}_{1} \\
\text { (Hytech) }\end{array}$ & $\begin{array}{c}\mathrm{V}_{2} \\
(\mathrm{Laxmi})\end{array}$ & $\begin{array}{c}\mathrm{V}_{3} \\
\text { (Mahalaxmi) }\end{array}$ & Mean \\
\hline $\mathrm{S}_{1}(\mathbf{1 . 5})$ & 11.53 & 10.50 & 10.44 & 10.82 & 12.52 & 11.51 & 10.79 & 11.61 & 11.69 & 10.92 & 10.49 & 11.03 \\
\hline $\mathrm{S}_{2}(\mathbf{5})$ & 10.35 & 9.47 & 8.98 & 9.60 & 11.44 & 11.02 & 10.44 & 10.97 & 10.06 & 10.01 & 9.93 & 10.00 \\
\hline $\mathrm{S}_{3}(\mathbf{8})$ & 8.49 & 8.11 & 7.40 & 8.00 & 9.45 & 9.17 & 9.09 & 9.24 & 9.83 & 8.35 & 8.16 & 8.78 \\
\hline $\mathrm{S}_{4}(12)$ & 7.87 & 7.12 & 6.36 & 7.12 & 8.93 & 8.90 & 8.44 & 8.76 & 8.07 & 7.92 & 7.89 & 7.96 \\
\hline Mean & 9.56 & 8.80 & 8.30 & & 10.59 & 10.15 & 9.69 & & 9.91 & 9.30 & 9.12 & \\
\hline & SEm \pm & $\begin{array}{l}\text { CD } \\
(\mathbf{0 . 0 5})\end{array}$ & \multicolumn{2}{|l|}{ CV\% } & SEm \pm & $\begin{array}{l}\text { CD } \\
(\mathbf{0 . 0 5})\end{array}$ & \multicolumn{2}{|l|}{ CV\% } & SEm \pm & $\begin{array}{c}\text { CD } \\
(\mathbf{0 . 0 5})\end{array}$ & \multicolumn{2}{|l|}{ CV\% } \\
\hline Factor $\mathbf{S}$ & 0.07 & 0.20 & \multirow{3}{*}{\multicolumn{2}{|c|}{2.27}} & 0.10 & 0.31 & \multirow{3}{*}{\multicolumn{2}{|c|}{3.10}} & 0.12 & 0.34 & \multirow{3}{*}{\multicolumn{2}{|c|}{3.68}} \\
\hline Factor V & 0.06 & 0.17 & & & 0.09 & 0.27 & & & 0.10 & 0.29 & & \\
\hline S X V & 0.12 & 0.34 & & & 0.18 & 0.53 & & & 0.20 & 0.58 & & \\
\hline
\end{tabular}

Table.4 Soil actinomycete population $\left(\mathrm{CFU} \mathrm{g} \mathrm{g}^{-1} \times 10^{4}\right)$ as influenced by salinity and sorghum cultivars

\begin{tabular}{|c|c|c|c|c|c|c|c|c|c|c|c|c|}
\hline \multirow{3}{*}{ 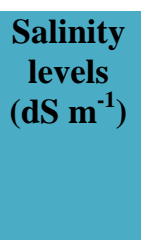 } & \multicolumn{4}{|c|}{$\begin{array}{l}\text { Soil actinomycete population }\left(\mathrm{CFU} \mathrm{g}^{-1} \times \mathbf{1 0}^{4}\right) \\
\text { at } 30 \text { DAS }\end{array}$} & \multicolumn{4}{|c|}{$\begin{array}{c}\text { Soil actinomycete population }\left(\mathrm{CFU} \mathrm{g}^{-1} \times \mathbf{1 0}^{4}\right) \\
\text { at flowering stage }\end{array}$} & \multicolumn{4}{|c|}{$\begin{array}{c}\text { Soil actinomycete population }\left(\mathrm{CFU} \mathrm{g}^{-1} \times \mathbf{1 0}^{4}\right) \\
\text { at harvest stage }\end{array}$} \\
\hline & \multicolumn{4}{|c|}{ Sorghum Cultivars } & \multicolumn{4}{|c|}{ Sorghum Cultivars } & \multicolumn{4}{|c|}{ Sorghum Cultivars } \\
\hline & $\begin{array}{c}\mathrm{V}_{1} \\
\text { (Hytech) }\end{array}$ & $\begin{array}{c}\mathrm{V}_{2} \\
(\text { Laxmi) }\end{array}$ & $\begin{array}{c}\mathbf{V}_{3} \\
\text { (Mahalaxmi) }\end{array}$ & Mean & $\begin{array}{c}\mathrm{V}_{1} \\
\text { (Hytech) }\end{array}$ & $\frac{\mathrm{V}_{2}}{(\text { Laxmi) }}$ & $\begin{array}{c}\mathbf{V}_{3} \\
\text { (Mahalaxmi) }\end{array}$ & Mean & $\begin{array}{c}\mathrm{V}_{1} \\
\text { (Hytech) }\end{array}$ & $\frac{\mathrm{V}_{2}}{(\text { Laxmi) }}$ & $\begin{array}{c}\mathrm{V}_{3} \\
\text { (Mahalaxmi) }\end{array}$ & Mean \\
\hline$S_{1}(1.5)$ & 15.25 & 15.04 & 14.80 & 15.03 & 17.15 & 17.03 & 16.96 & 17.04 & 16.00 & 15.33 & 14.74 & 15.35 \\
\hline $\mathrm{S}_{2}(5)$ & 14.16 & 13.87 & 13.60 & 13.88 & 14.97 & 14.90 & 14.81 & 14.89 & 14.59 & 14.35 & 13.85 & 14.26 \\
\hline$S_{3}(8)$ & 11.70 & 11.20 & 11.05 & 11.32 & 13.00 & 12.92 & 12.83 & 12.92 & 12.24 & 11.96 & 11.32 & 11.84 \\
\hline$S_{4}(12)$ & 9.68 & 9.04 & 8.78 & 9.17 & 11.46 & 11.31 & 10.79 & 11.19 & 11.23 & 10.90 & 10.61 & 10.91 \\
\hline \multirow[t]{2}{*}{ Mean } & 12.70 & 12.29 & 12.06 & & 14.15 & 14.04 & 13.85 & & 13.51 & 13.13 & 12.63 & \\
\hline & SEm \pm & $\begin{array}{c}\text { CD } \\
(\mathbf{0 . 0 5})\end{array}$ & \multicolumn{2}{|l|}{ CV\% } & SEm \pm & $\begin{array}{c}\text { CD } \\
(\mathbf{0 . 0 5})\end{array}$ & \multicolumn{2}{|l|}{ CV\% } & SEm \pm & $\begin{array}{c}\text { CD } \\
(\mathbf{0 . 0 5})\end{array}$ & \multicolumn{2}{|l|}{ CV\% } \\
\hline Factor $\mathrm{S}$ & 0.04 & 0.12 & \multirow{3}{*}{\multicolumn{2}{|c|}{1.01}} & 0.05 & 0.15 & \multirow{3}{*}{\multicolumn{2}{|c|}{1.07}} & 0.05 & 0.15 & \multirow{3}{*}{\multicolumn{2}{|c|}{1.17}} \\
\hline $\begin{array}{c}\text { Factor } \\
\text { V }\end{array}$ & 0.04 & 0.11 & & & 0.04 & 0.13 & & & 0.04 & 0.13 & & \\
\hline S X V & 0.07 & 0.21 & & & 0.09 & 0.25 & & & 0.09 & 0.26 & & \\
\hline
\end{tabular}


Table.5 Soil dehydrogenase enzyme activity $\left(\mu \mathrm{g} \mathrm{TPF}^{-1}\right.$ day $\left.^{-1}\right)$ as influenced by salinity and sorghum cultivars

\begin{tabular}{|c|c|c|c|c|c|c|c|c|c|c|c|c|}
\hline \multirow{3}{*}{$\begin{array}{l}\text { Salinity } \\
\text { levels } \\
\left(\mathrm{dS} \mathrm{m}^{-1}\right)\end{array}$} & \multicolumn{4}{|c|}{$\begin{array}{l}\text { Soil dehydrogenase enzyme activity } \\
\qquad\left(\mu \mathrm{g} \mathrm{TPF}^{-1} \text { day }^{-1}\right) \text { at } 30 \text { DAS }\end{array}$} & \multicolumn{4}{|c|}{$\begin{array}{l}\text { Soil dehydrogenase enzyme activity } \\
\left(\mu \mathrm{g} \mathrm{TPF}^{-1} \text { day }^{-1}\right) \text { at flowering stage }\end{array}$} & \multicolumn{4}{|c|}{$\begin{array}{l}\text { Soil dehydrogenase enzyme activity } \\
\left(\mu \mathrm{g} \text { TPF } \mathrm{g}^{-1} \text { day }^{-1}\right) \text { at harvest stage }\end{array}$} \\
\hline & \multicolumn{4}{|c|}{ Sorghum cultivars } & \multicolumn{4}{|c|}{ Sorghum cultivars } & \multicolumn{4}{|c|}{ Sorghum cultivars } \\
\hline & $\begin{array}{c}\mathrm{V}_{1} \\
\text { (Hytech) }\end{array}$ & $\begin{array}{c}\mathrm{V}_{2} \\
\text { (Laxmi) }\end{array}$ & $\begin{array}{c}\mathbf{V}_{3} \\
\text { (Mahalaxmi) }\end{array}$ & Mean & $\begin{array}{c}\mathrm{V}_{1} \\
\text { (Hytech) }\end{array}$ & $\begin{array}{c}\mathrm{V}_{2} \\
\text { (Laxmi) }\end{array}$ & $\begin{array}{c}\mathrm{V}_{3} \\
\text { (Mahalaxmi) }\end{array}$ & Mean & $\begin{array}{c}\mathrm{V}_{1} \\
\text { (Hytech) }\end{array}$ & $\begin{array}{c}\mathrm{V}_{2} \\
\text { (Laxmi) }\end{array}$ & $\begin{array}{c}\mathrm{V}_{3} \\
\text { (Mahalaxmi) }\end{array}$ & Mean \\
\hline $\mathrm{S}_{1}(\mathbf{1 . 5})$ & 15.63 & 13.96 & 12.77 & 14.12 & 29.48 & 28.28 & 25.28 & 27.68 & 21.82 & 21.32 & 21.23 & 21.46 \\
\hline$S_{2}(5)$ & 10.92 & 9.61 & 8.46 & 9.66 & 25.84 & 25.28 & 24.98 & 25.36 & 21.44 & 19.42 & 16.22 & 19.03 \\
\hline $\mathrm{S}_{3}(\mathbf{8})$ & 7.61 & 7.21 & 6.81 & 7.21 & 19.90 & 19.32 & 18.95 & 19.39 & 14.74 & 14.42 & 13.92 & 14.36 \\
\hline $\mathrm{S}_{4}(12)$ & 5.18 & 4.00 & 3.00 & 4.06 & 16.80 & 16.46 & 16.15 & 16.47 & 11.04 & 10.77 & 10.27 & 10.69 \\
\hline Mean & 9.84 & 8.70 & 7.76 & & 23.00 & 22.33 & 21.34 & & 17.26 & 16.48 & 15.41 & \\
\hline & SEm \pm & $\mathrm{CD}(0.05)$ & CV\% & & SEm \pm & $\mathrm{CD}(0.05)$ & CV\% & & SEm \pm & CD (0.05) & CV\% & \\
\hline Factor $\mathrm{S}$ & 0.10 & 0.30 & 3.57 & & 0.29 & 0.86 & 3.98 & & 0.20 & 0.59 & 3.67 & \\
\hline Factor V & 0.09 & 0.26 & & & 0.26 & 0.74 & & & 0.17 & 0.51 & & \\
\hline S X V & 0.18 & 0.53 & & & 0.51 & 1.49 & & & 0.35 & 1.01 & & \\
\hline
\end{tabular}


Several root exudates are the carbon compounds like sugars, amines etc which act as food for organisms.

Study conducted by Matsuguchi and Sakai (1995) at different salinity levels revealed that all microbial populations may not be affected similarly by salinity stress. Some increase in number while, others follow a declining trend. The detrimental effect of salinity on the activity of microbial population (bacteria, fungi and actinomycetes) could be due to the manipulation of growth of living cells either by disrupting the normal physiological activities or intracellular macromolecular structures (Vogel et al., 2010). The decline in microbial population could also be due to increase in salt concentration, which decreased osmotic potential and lead to reduction in activity of surviving cells (Ibekwe et al., 2010). Similar results were reported by Wichern et al., (2006).

The high influence of salinity on fungi was also reported earlier and was attributed to genetic diversity (Van and Semenov, 2000) and cell damage by the low osmotic potential (Hagemann, 2011).

\section{Dehydrogenase enzyme activity}

Data pertaining to soil dehydrogenase activity measured at different growth stages of crop viz., 30 DAS, flowering and harvest as affected by salinity levels in different sorghum cultivars are presented in table 5 . The soil dehydrogenase activity was significantly influenced by the salinity levels, varieties and their interaction.

In general the dehydrogenase activity decreased with increasing salt concentration and degree of reduction varied with the salinity levels. At 30 DAS, flowering and at harvest the highest dehydrogenase activity of 14.12, 27.68 and $21.46 \mu \mathrm{g}$ TPF $\mathrm{g}^{-1}$ day $^{-1}$, respectively was recorded at the lowest salinity level of $1.5 \mathrm{dS} \mathrm{m} \mathrm{m}^{-1}$, which was found to be significant over the remaining salinity levels. The lowest dehydrogenase activity of 4.06, 16.47 and 10.69 $\mu \mathrm{g}$ TPF g ${ }^{-1}$ day $^{-1}$, was recorded at higher salinity level of $12 \mathrm{dS} \mathrm{m}^{-1}$ at 30 DAS flowering and at harvest, respectively. At all the three stages each level was significantly superior over the higher levels of salinity. From the data, it can be inferred that the per cent reduction in soil dehydrogenase activity from the lowest salinity level to the highest salinity level was $71.25,40.50$ and 50.19 at $30 \mathrm{DAS}$, flowering and harvest, respectively.

At all stages of crop growth the highest dehydrogenase activity of 9.84, 23.00 and $17.26 \mu \mathrm{g}$ TPF $\mathrm{g}^{-1}$ day $^{-1}$ was observed with cultivar Hytech whereas, the lowest dehydrogenase activity of 7.76, 21.34 and $15.41 \mu \mathrm{g}$ TPF $\mathrm{g}^{-1}$ day $^{-1}$ was observed with cultivar Mahalaxmi but at flowering the highest dehydrogenase activity of $23.00 \mu \mathrm{g}$ TPF $\mathrm{g}^{-1}$ day $^{-1}$ was observed with cultivar Hytech. The sorghum cultivar Hytech, maintained the higher dehydrogenase activity at all stages of the crop growth whereas, the lowest was recorded by the cultivar Mahalaxmi at all stages of the crop growth.

The interactions revealed that the highest dehydrogenase activity was recorded in treatment combination $1.5 \mathrm{dS} \mathrm{m}^{-1} \mathrm{x}$ Hytech at all growth stages and all other treatment combinations were inferior to $1.5 \mathrm{dS} \mathrm{m}^{-1} \mathrm{x}$ Hytech. At all stages the lowest was recorded with Mahalaxmi at the highest salinity $12 \mathrm{dS}$ $\mathrm{m}^{-1} \mathrm{x}$ Mahalaxmi.

The decline in enzyme activity with increasing salinity appeared to be associated with change in osmotic potential of the soil due to higher salt concentrations, specific ion toxicities and salting out effect of soluble salts on enzyme protein (Iftikhar and Khan, 1988). 
An excessive content of easily-soluble inorganic salts in soil has a negative effect on the changes in the conformation of enzymatic protein which results in a decrease in its catalytic activity (Siddikee et al., 2011).

Salinity levels and sorghum cultivars affected the soil biological activity. Soils of high salinity (12 $\mathrm{dS} \mathrm{m}^{-1}$ ) were deprived of all the biological properties of soil. The cultivar Hytech maintained better soil properties at all stages of crop growth compared to Laxmi and Mahalaxmi.

\section{References}

Casida, L., Klein, D and Santoro, T. 1964. Soil dehydrogenase activity. Soil Science. 98: 371-376.

Hagemann, M. 2011. Molecular biology of cyanobacterial salt acclimation. Fems Microbiology Reviews. 35(1): 87-123.

Ibekwe, A., Poss, J.A., Grattan, S.R., Grieve, C.M and Suarez, D. 2010. Bacterial diversity in cucumber (Cucumis sativus) rhizosphere in response to salinity, soil $\mathrm{pH}$ and boron. Journal of Soil Biology and Biochemistry. 42: 567-575.

Iftikhar, A and Khan, K.M. 1988. Studies on enzyme activity in normal and saline soils. Pakistan Journal of Agricultural Research. 9: 505-508.

Jackson, M.L. 1973. Soil Chemical Analysis, Prentice Hall India Private Limited, New Delhi: 41.

Jiang, H., Dong, H., Yu, B., Liu, X., Li, Y., Ji, $S$ and Zhang, C.L. 2007. Microbial response to salinity change in Lake Chaka, a hypersaline lake on Tibetan plateau. Environmental Microbiology. 9(10): 2603-2621.

Matsuguchi, T and Sakai, M. 1995. Influence of soil salinity on the populations and composition of fluorescent pseudomonads in plant rhizosphere. Soil
Science and Plant Nutrition. 41(3): 497504.

Oren, A. 1999. Bioenergetic aspects of halophilism. Microbiology and Molecular Biology Reviews. 65: 334348.

Paroda, K.K. 2007. Experimental Soil Microbiology. CBS publishers and distributors, New Delhi. 9-18.

Pathak, H. and D.L.N. Rao. 1998. Carbon and nitrogen mineralization from added organic matter in saline and alkali soils. Soil Biology and Biochemistry. 30: 695702.

Petersen, F.H. 1996. Water Testing and Interpretation. In: Reed, D.W. (ed.) Water, media and nutrition for greenhouse crops. Batavia: Ball. cap.2, pp.31-49.

Rietz, D.N. and R.J. Haynes. 2003. Effect of irrigation-induced salinity and sodicity on soil microbial activity. Soil Biology and Biochemistry. 35: 845-854.

Rietz, D.N., R.J. Haynes and S. Chidoma. 2001. Effect of soil salinity induced under irrigated sugarcane in the Zimbabwean lowland on soil microbial activity. South African Sugar Technologist's Association. 75: 68-74.

Siddikee, M.A., Tipayno, S.C., Kim, K., Chung, J.B and Sa, T. 2011. Influence of varying degree of salinity-sodicity stress on enzyme activities and bacterial populations of coastal soils of Yellow Sea, South Korea. Journal of Microbiology and Biotechnology. 2: 341-346.

Tripathi, S., Kumari, S., Chakraborty, A., Gupta, A., Chakrabarti, K and Bandyapadhyay, B.K. 2006. Microbial biomass and its activities in saltaffected soils. Biology and Fertility of Soils. 42(3): 273-277.

Van, B.A.H.C and Semenov, A.M. 2000. In search of biological indicators for soil 
health and disease suppression. Applied Soil Ecology. 15(1): 13-24.

Vogel, B.F., Hansen, L.T., Mordhorst, H and Gram, L. 2010. The survival of Listeria monocytogenes during long term desiccation is facilitated by sodium chloride and organic material. International Journal of Food Microbiology. 140(2-3): 192-200.
Wichern, J., Wichern, F and Joergensen, R.G. 2006. Impact of salinity on soil microbial communities and the decomposition of maize in acidic soils. Geoderma. 137(1\&2): 100-108.

Zahran, Z. (1997). Diversity, adaptation and activity of the bacterial flora in saline environments. Biology and Fertility of Soils. 25(3): 211-223.

\section{How to cite this article:}

Adilakshmi, K., P. Prasuna Rani, P. Ratna Prasad and Lakshmipathy, R. 2018. Biological Properties of Saline Soils Grown with Different Sorghum Cultivars. Int.J.Curr.Microbiol.App.Sci. 7(04): 2096-2105. doi: https://doi.org/10.20546/ijcmas.2018.704.240 